\title{
Diabète insulinodépendant et HLA-DQ : un modèle moléculaire de susceptibilité
}

Un modèle moléculaire est proposé pour le diabète insulinodépendant (DID). Il fait jouer un rôle important à deux régions diamétralement opposées, aux deux extrémités de la cavité engendrée par les chaînes $\alpha$ et $\beta$ de la molécule HLA-DQ. Les différentes combinaisons moléculaires sont fondées sur la présence ou l'absence de deux marqueurs : l'un susceptible (Arg 52 DQ $\alpha$ ), nouvellement décrit, l'autre protecteur (Asp $57 \mathrm{DQ} \beta)$, précédemment connu. Ainsi la susceptibilité à la maladie n'est pas régie par une loi du "tout ou rien " mais par une gamme de degrés de risque suivant la densité de ces molécules, la présence d'Arg $52 \mathrm{DQ} \alpha$ et l'absence d'Asp 57 DQ $\beta$ conférant la susceptibilité.

\section{Iman Khalil}

Virginia Lepage

Laurent Degos

Francis Galibert

Ingeborg Deschamps Jacques Hors

\section{ADRESSES}

I. Khalil: chargé de recherche, Inserm U.93. V. Lepage : praticien hospitalier. L. Degos : professeur à l'université Paris VII, directeur de l'U.93 de l'Inserm. J. Hors : professeur à l'université Paris VII. F. Galibert : directeur, Cnrs $U P R$ 41. Hôpital Saint-Louis, 1 av. C.Vellefaux, 75010 Paris, France. I. Deschamps : chargée de recherche, Inserm U.30. Hôpital des Enfants-Malades, 149, rue de Sèvres, 75010 Paris, France.

$\mathrm{m} / \mathrm{s} \mathrm{n}^{\circ} 3$, vol. 7 mars 9

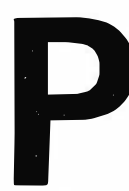

armi les différents gènes du système HLA situés sur le bras court du chromosome 6 , ceux qui codent pour les molécules de classe II (DR, DQ, DP) jouent un rôle crucial dans la présentation du peptide, étape initiale de la réponse immune. Ces molécules sont particulièrement polymorphes. Elles joueraient un rôle important dans la susceptibilité aux maladies autoimmunes.

Dans le groupe des maladies associées ou liées au système HLA [1], le diabète insulinodépendant (DID) présente deux particularités : le risque maximal correspond à la coexistence de deux allèles différents HLA de classe II DR3/DR4 (susceptibilité) alors qu'au contraire un autre allèle, DRw15, semble avoir un effet protecteur puisqu'il est pratiquement absent chez les malades.

\section{Les marqueurs de la susceptibilité}

HLA-DR. Les études des dix dernières années ont montré que 90 à
$95 \%$ des malades diabétiques (DID de type 1) sont HLA-DR3 et/ou DR4. Le risque relatif de DID est assez faible pour les sujets possédant soit HLA-DR3 soit DR4, comme pour ceux qui possèdent ces mêmes antigènes à l'état homozygote (DR3/DR3 ou DR4/DR4). En revanche, les sujets possédant les deux marqueurs à l'état hétérozygote (DR3/DR4) ont un risque très augmenté (risque relatif ou RR $=47$ ) . Ainsi $30 \%$ des malades atteints de DID possèdent cette combinaison contre $1 \%$ dans la population normale [2]. Cette observation suggère que la susceptibilité est le résultat non pas d'un seul, mais de deux facteurs génétiques agissant de manière complémentaire.

HLA-DQ. L'avènement des techniques de biologie moléculaire a permis une définition plus fine des spécificités en cause et a démontré l'importance prépondérante des allèles DQ dont les loci (DQA et DQB codant pour les chaînes $\alpha$ et $\beta$ des molécules $\mathrm{DQ}$ ) sont voisins des loci $\mathrm{DR}$ et en très fort déséquilibre de liaison avec eux. 


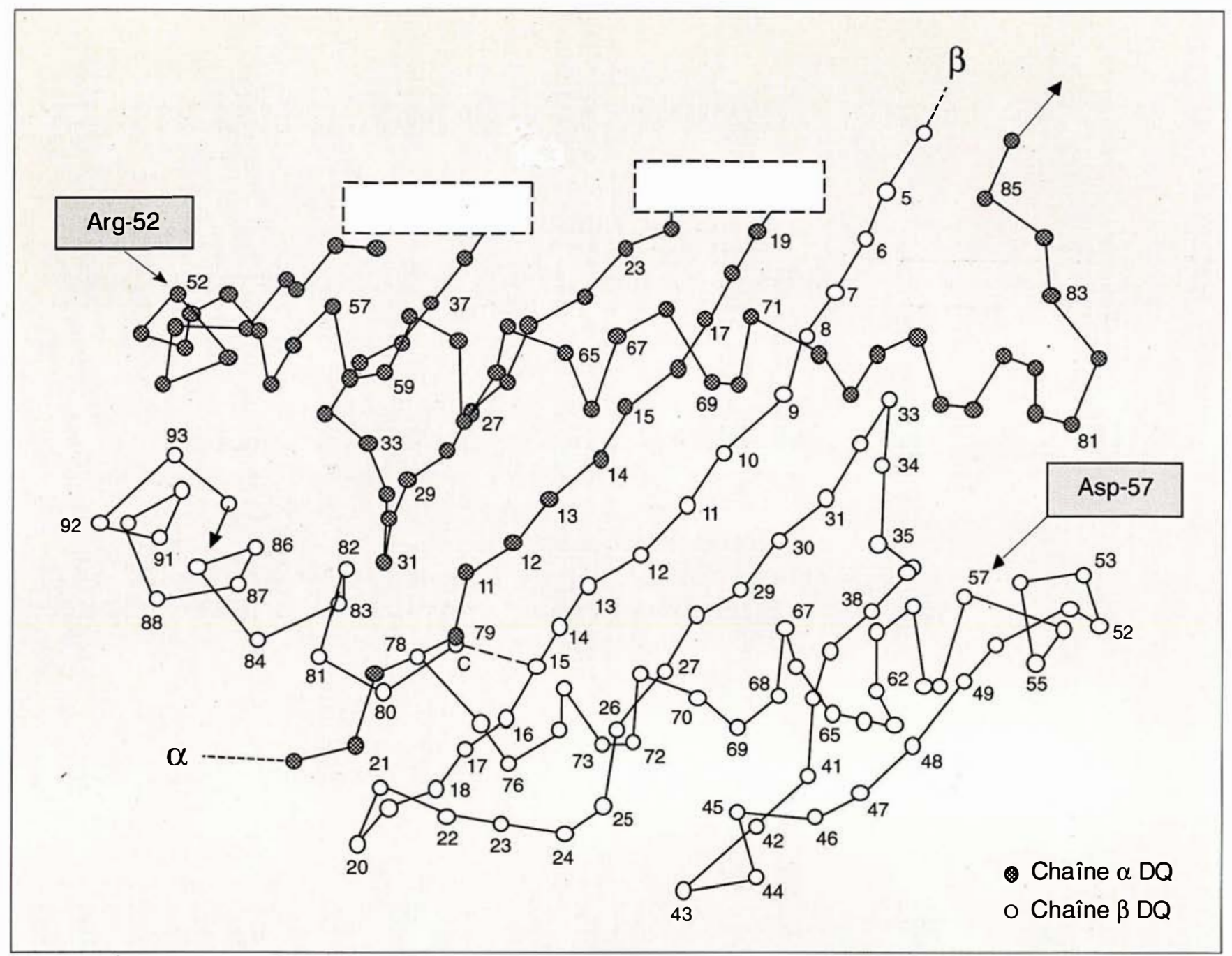

Figure 1. Structure tridimensionnelle postulée de la molécule HLA-DQ (d'après [7]. Les flèches indiquent la localisation des deux résidus Arg-52 (DQ $\alpha)$ et Asp-57 (DQß).

La technique de fragments de restrictions polymorphes (restriction fragment length polymorphisms, $\mathrm{m} / \mathrm{s}$ supplément au $n^{\circ} 7$, vol 3, p. 4) a permis de distinguer, parmi les spécificités DQ classiques et définies par un typage sérologique $\left(\mathrm{m} / \mathrm{s}\right.$ supplément au $n^{\circ} 1$, vol 5 , p. 22), plusieurs sous-types dont certains seulement semblent conférer une susceptibilité à la maladie. Par exemple, DR4 peut s'associer aux allèles DQw4, w7 ou w8; mais l'haplotype DR4-DQw8 est retrouvé chez la quasi-totalité (plus de $90 \%$ ) des malades diabétiques DR4 positifs alors qu'il n'est présent que chez 60 à $65 \%$ de la population générale.
La détermination des séquences nucléotidiques a permis à Todd et al. [3] de démontrer que la susceptibilité au DID est corrélée à l'absence de l'acide aspartique en position 57 de la chaîne HLA-DQ $\beta$. Cependant, tous les haplotypes DQB Asp 57 négatifs ne confèrent pas la susceptibilité. Par exemple, les haplotypes HLA-DR3 et HLA-DR7 sont tous deux en déséquilibre de liaison avec la spécificité HLA-DQw2 qui est HLA-DQ $\beta$ Asp 57 négatif. Or, HLA-DR3-DQw2 est fortement associé au DID alors que HLADR7-DQw2 ne l'est pas.

De plus, l'analyse de séquences des allèles HLA-DQ associés à HLADR7, et DRw9 chez les malades diabétiques d'ethnies noires, a révélé qu'ils possèdent la même chaîne $\mathrm{DQ} \alpha$ que celle des malades caucasoides porteurs de HLA-DR4-DQw8. Cela suggère que d'autres gènes que DQB - et notamment HLA-DQA - pourraient être impliqués dans la susceptibilité.

Pour évaluer le rôle respectif de HLA-DQA et DQB, nous avons effectué un typage exhaustif utilisant la technique d'amplification par la Taq polymérase (PCR) suivie d'une hybridation spécifique [4]. Cela nous a permis d'identifier 8 allèles DQA et 
12 allèles DQB. Par l'évaluation des fréquences alléliques de 50 malades et 73 témoins et par la comparaison de séquences correspondantes, il a été mis en évidence un marqueur de susceptibilité localisé sur la chaîne HLA$\mathrm{DQ} \alpha$. Il s'agit de la présence de l'acide aminé arginine en position 52 [5].

\section{Modèle moléculaire de la susceptibilité}

Le modèle que nous proposons est fondé sur les conceptions nouvelles de la structure des molécules de classe II, récepteurs de l'antigène [6]. Ces molécules sont en effet des hétérodimères formés d'une chaîne $\alpha$ et d'une chaîne $\beta$ dont les domaines extérieurs (deux hélices $\alpha$ ) se font face, bordant une profonde cavité, site de liaison du peptide étranger de l'auto-antigène. Les régions qui sont au contact de l'antigène présentent une grande variabilité, et les acides aminés présents en certaines positions de ces régions, ainsi que leur charge (figure 1), pourraient être déterminants pour la sélection du ou des peptides effectivement liés et exposés à la surface des cellules présentatrices de l'antigène [7].

Les positions $\mathrm{DQ} \alpha 52$ et $\mathrm{DQ} \beta 57$ sont localisées aux deux extrémités de la molécule $\mathrm{DQ}$. Le modèle que nous proposons postule que la susceptibilité au DID est fondée sur la formation d'une molécule $\mathrm{DQ}$ composée de chaînes $\alpha$ et $\beta$ "susceptibles " (SS, figure 2), c'est-à-dire caractérisées par l'absence de résidu aspartique en position DQ $\beta 57$ et la présence d'une arginine en position $\mathrm{DQ} \alpha 52$.

A l'inverse, la combinaison d'une chaîne DQ $\alpha 52$ Arg - et d'une chaîne DQ $\beta 57$ Asp + engendre une molécule " protectrice " (PP). Les molécules formées d'une chaîne susceptible et d'une chaîne protectrice (SP ou PS, figure 2) sont supposées protectrices.

Il est important de noter ici que les molécules hétérodimères $\mathrm{DQ}$ peuvent se former soit en cis (les chaînes $\alpha$ et $\beta$ sont codées par des gènes DQA et DQB situés sur le même chromosome), soit en trans (les chaînes $\alpha$ et $\beta$ sont codées par des gènes de chromosomes différents). Par conséquent, chaque individu a la possibilité théo-

$\mathrm{m} / \mathrm{s} n^{\circ} 3$, vol. 7 , mars 91 rique d'exprimer quatre molécules DQ différentes, issues des combinaisons en cis ou en trans des produits codés par les gènes DQA et DQB des deux haplotypes qu'il possède (figure 3). Dans le cas, par exemple, d'un individu possédant sur un haplotype $\mathrm{DQ} \alpha 52 \mathrm{Arg}+\mathrm{DQ} \beta$ $57 \mathrm{Asp}+$, et sur l'autre $\mathrm{DQ} \alpha$ $52 \mathrm{Arg}^{-} \mathrm{DQ} \beta 57 \mathrm{Asp}^{-}$, on peut donc s'attendre à ce qu'il exprime quatre types différents de molécules $\mathrm{DQ}$ : SP et PS en cis, SS et PP en trans (3e colonne de la figure 3 , p. 238).

Dans l'échantillon que nous avons étudié, tous les malades ont en commun la possibilité de former une molécule "SS " que ce soit en cis ou en trans. De plus, le degré de susceptibilité à la maladie semble fonction du nombre de molécules "susceptibles " ou " protectrices " qu' un individu est capable d'exprimer compte tenu des gènes $\mathrm{DQA}$ et $\mathrm{DQB}$ présents sur ses deux haplotypes. A partir des 4 combinaisons ( 2 en cis, et 2 en trans), la proportion des molécules SS, SP ou PS et PP paraît corrélée à la susceptibilité au diabète (figure 3).

Ainsi, $64 \%$ des malades se trouvent dans la situation de pouvoir exprimer uniquement des molécules DQ de type SS. L'absence de sujets normaux dans ce cas est probablement due à à un effet d'échantillonnage. On connaît, en effet, au moins une catégorie de sujets "à risque maximal " qui pourtant ne sont pas toujours atteints : il s'agit des frères ou sœurs HLA identiques à des malades

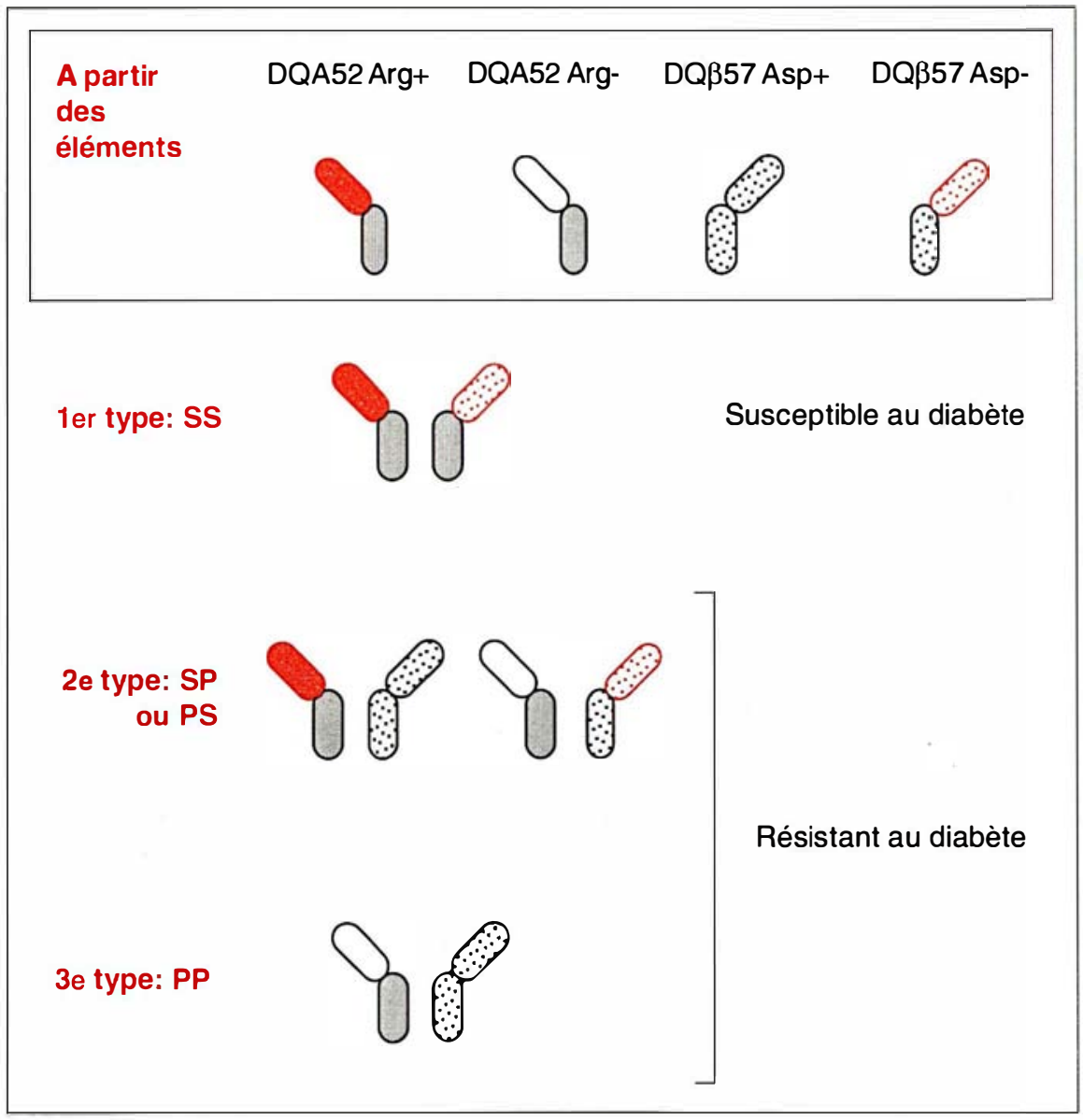

Figure 2. Types de molécules DQ à la surface cellulaire. 


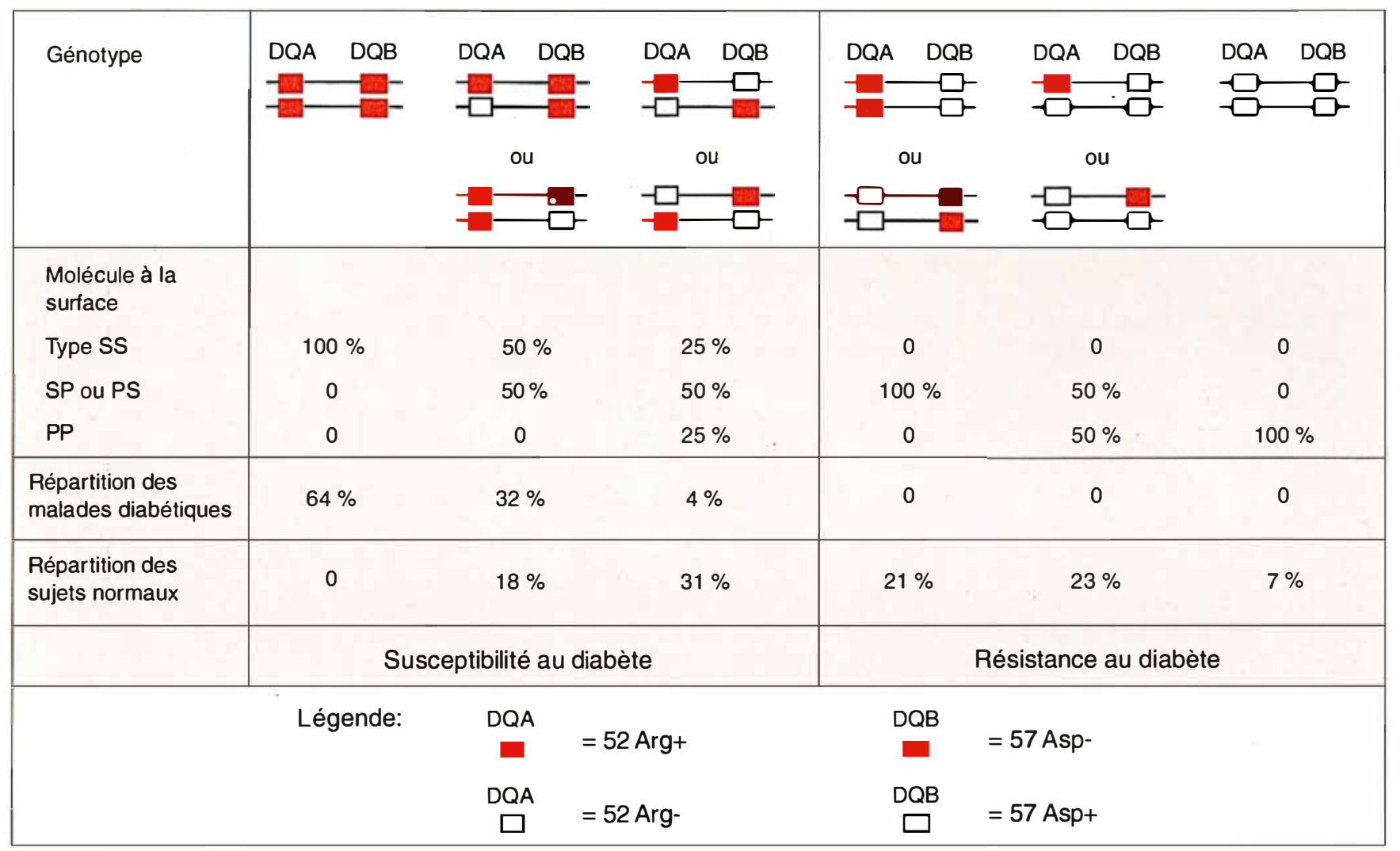

Figure 3. Prédiction du risque en fonction des molécules exprimées à partir du génotype.

\section{RÉFÉRENCES}

1. Hors J. In : Dousset J, Pla M, ed. HLA et maladies. HLA complexe majeur d'histocompatibilité de l'homme. Paris: Flammarion, 1989 : 298-316.

2. Deschamps I, Lestradet H, MarcelliBarge A, Poirier JC, Cohen D, Hors J. Facteurs de risque du diabète insulinodépendant. Pathol Biol 1986; 34 : 767-72.

3. Todd JA, Bell Jl, McDevitt HO. HLA$\mathrm{DQ} \beta$ gene contributes to susceptibility and resistance to insulin-dependent diabetes mellitus. Nature 1987 ; 329 : 599-604.

4. Khalil I, d'Auriol L, Morin L, et al. Typing for HLA-DQ using oligonucleotidic probes. Nouv Rev Fr Hematol 1990 ; 32 109-12.

5. Khalil I, d'Auriol L, Gobet M, et al. A combination of HLA-DQ $\beta$ Asp 57 negative lity to insulin-dependent diabetes mellitus. J Clin Invest 1990; 85 : 1315-19.

6. Brown JH, Jardetzky MA, Saper B, Samraoui P, Bjorkman PJ, Wiley DC. A hypothetical model of the foreign antigen binding site of class II histocompatibility molecules. Nature 1988 ; 332 : 845-50.

7. Claverie JM. Immunologie 1989 : la révolution peptidique. médecine/sciences 1989 . $6: 367-76$

8. Todd JA, Fukui Y, Kitagawa T, Sasazuki $\mathrm{T}$. The A3 allele of the HLA-DQA 1 locus is associated with susceptibility to type 1 diabetes in Japanese. Proc Natl Acad Sci USA 1990 ; 87 : 1094-98.

9. Feutren G, Assan R, Karsenty G, et al. Cyclosporin increases the rate and length of remissions in insulin-dependant diabetes of recent onset. Results of a multicentre double blind trial. Lancet 1986; ii : 119-24.
DR3/DR4. Cependant, les trois quarts de ceux-là ne seront jamais diabétiques, suggérant l'existence d'autres facteurs génétiques ou environnementaux. Un second groupe de malades $(32 \%)$ est constitué de sujets qui ont des chances équivalentes d'exprimer des molécules susceptibles (SS) et résistantes (SP ou PS). Seulement deux malades (4\%) se trouvent dans une situation où la majorité (3 sur 4 ) des molécules DQ est de type protecteur (SP, PS ou PP) ; dans ce cas, cependant, un quart des hétérodimères reste de type SS. Aucun malade n'a été répertorié dans le groupe des individus n'exprimant pas de molécules SS.

Nous ignorons encore aujourd'hui les mécanismes de l'effet protecteur. Peut-être les molécules DQ $\alpha 52$ 
Arg + /DQ $\beta 57$ Asp - permettent-elles une présentation particulièrement efficace de peptides correspondant à des auto-antigènes des cellules $\beta$ des îlots de Langerhans, ou bien à des antigènes viraux ? Ce seraient alors les résidus aux positions 52 de la chaîne $\alpha$ et 57 de la chaîne $\beta$, ou bien des acides aminés environnants, qui permettraient une telle interaction élective avec certains peptides.

Le modèle que nous proposons doit encore être vérifié dans d'autres ethnies. Il faut noter que le rôle important de la chaîne $\mathrm{DQ} \alpha$ est dé jà confirmé par des observations récentes [8] portant sur des individus japonais dont beaucoup possèdent le résidu $\mathrm{DQ} \beta$ Asp57 " protecteur " et sont pourtant atteints de diabète.

\section{Médecine}

\section{prédictive-préventive}

Le modèle proposé permet de mieux définir et de dénoncer le risque de diabète suivant une échelle à plusieurs niveaux. Il suggère un mécanisme moléculaire d'affinité pour un peptide encore hypothétique qui serait anormalement présenté aux mécanismes inducteurs de la réponse immune.

Pour la première fois, un risque relatif dépassant la valeur de 100 est proposé pour le DID : $100 \%$ des malades portent la combinaison SS contre 36/73 (49\%) chez les individus normaux. C'est le seuil empiriquement accepté pour l'indication d'un dépistage d'intérêt prédictif.

Il est certain, cependant, que les facteurs génétiques ne déterminent la susceptibilité au DID que pour une part, le risque étant dû, pour moitié, à des facteurs aléatoires environnementaux.

Il est clair aussi que la prédiction doit s'intégrer dans une éthique médicale nullement passive mais bien au contraire étroitement associée à un essai de prévention, comme cela a été réalisé dans le passé [9]

\section{Remerciements}

Nous tenons à remercier Hélène Bleux pour la préparation du manuscrit. Ce travail est réalisé dans le cadre de GEOD, projet MRT $88 \mathrm{~T} 0845$.

\section{Summary}

Insulin dependent diabetes mellitus and HLA-DQ : a molecular model of susceptibility

Many contributions have brought evidence that the highest risk of Insulin Dependent Diabetes Mellitus (IDDM) is associated, at least in caucasians, with the heterozygous combination HLADR3/DR4. Todd et al. have reported that the susceptibility to the disease correlates with the HLA-DQ $\beta$ chain which is in linkeage disequilibrium with HLADR3 and DR4; more precisely with the absence of aspartic acid residue at position 57 of the $\mathrm{DQ} \beta$ chain (DQ $\beta$ Asp 57 negative). However, DS $\beta$ Asp 57 negative haplotypes do not always confer susceptibility and conversely, some $\mathrm{DQ} \beta$ Asp 57 positive haployypes seem to be disease-associated. Recently, we have extended this observation and postulated the probable implication of an another marker : an arginine residue at position 52 of the $\mathrm{DQ} \alpha$ chain. Moreover, a molecular model of susceptibility to IDDM has been proposed, based on the expression at the cell surface, in cis and trans, of a susceptible HLA-DQ heterodimer composed of a : DQ $\alpha$ Arg 52 positive/HLA DQ $\beta$ Asp 57 negative. All other $\mathrm{DQ} \alpha / \beta$ heterodimers are considered as protective molecules. Moreover, the degree of risk seemed to depend on the number of such " susceptible" DQ heterodimers that a subject may theoretically be able to express according to his HLADQA1 and DQB1 alleles.

NDLR. A propos de cet article, voir aussi le com mentaire de J.F. Bach, page 287 de ce numéro

\section{TIRÉS A PART}

